describe CTSI processes, participation, products, and outcomes. Program components responded differently to the collaborative approach implemented. The M\&E technical assistance was implemented in 3 different ways: components either did the M\&E RPPR template themselves, with minimal M\&E team assistance; responded to comments and information provided by the M\&E team as a first step; or requested a significant level of assistance from $M \& E$. Participants/partners in developing and using RPPR include CTSI program leadership and staff, administration, communication staff, M\&E team, and our collaborators. RESULTS/ANTICIPATED RESULTS: The proposed comprehensive approach to the annual program performance reporting shows sound promise to enhance program staff engagement, report utilization, learning, strategic management, self-evaluation capacity, and continuous improvement within a clinical and translational science organization. DISCUSSION/SIGNIFICANCE OF IMPACT: This structured approach's impact is significant in that it fills the current gap in the practice, literature, and methodology and offers a practical example of a "practice that works" for CTR (and other) organizations and programs striving to improve their reporting practices, staff engagement, learning, and program impact. Leveraging and synergizing the RPPR requirements and other complex, data-demanding obligations and needs can help the CTS programs move beyond the once-a-year compilation of project accomplishments and challenges to developing and sharing a thoughtful translational science program success story. References: National Center for Advancing Translational Sciences. (2016). NCATS Strategic Plan. NIH. Available at: https://ncats.nih.gov/strategicplan Smith, C., Baveja, R., Grieb, T., \& Mashour, G. (2017). Toward a science of translational science. Journal of Clinical and Translational Science, 1(4), 253-255. doi:10.1017/cts.2017.14

3428

Adopting a learning health system architecture: a scoping review and pre-implementation framework to reduce readmissions within academic hospitals

Jami Anderson ${ }^{1}$, Becky Reamy and Michael Mugavero

${ }^{1}$ University of Alabama at Birmingham

OBJECTIVES/SPECIFIC AIMS: Of the six Centers for Medicare and Medicaid Services (CMS) monitored diagnoses targeted for readmissions reductions, reasons for readmissions within academic hospitals are poorly understood and reflect complex interactions between the patient, provider and organizational-level responses to initial hospitalization. Learning health systems (the organizational and orchestrated integration of research into evidence-based practice) can address the complexities of readmissions through an innovative approach to knowledge translation and patient-centered outcomes research. The objective of this review is to define and optimize the architecture of learning health systems to produce a dynamic pre-implementation framework of knowledge translation and patient-centered outcomes research, leveraging two engines (research and learning) within the academic and clinical settings for reducing readmissions. METHODS/STUDY POPULATION: Three databases were utilized for this scoping review (PubMed, Academic Search Premier, and Scopus) focusing on 1.) learning health systems and the methods of defining and building these systems within an academic hospital setting and 2.) the use of learning health systems in reducing readmissions within academic hospitals. Empirical articles and reviews pertaining to the architecture, development, conceptualization, definition, and translation of learning health systems were identified and compiled into a scoping review and proposed framework. RESULTS/ANTICIPATED RESULTS: The scoping review yielded 139 articles; from which 28 articles were retained. No articles were found utilizing learning health systems to address readmissions. Thus, a new architectural framework was developed incorporating common architectural themes from the literature with adaptations to fit the interests of patients, providers, and researchers in reducing readmissions within academic hospitals (Figure 1). DISCUSSION/SIGNIFICANCE OF IMPACT: Given the dearth of information applying learning health systems to readmissions, the proposed architecture for an integrative learning health system can be utilized as a dynamic foundation for adoption and pre-implementation planning for reducing readmissions within academic hospital settings. Additionally, the authors expect this model to be tested and continually refined to address historical and emerging issues for clinically-relevant and clinically-effective approaches to patient-centered practice and research.

3255

\section{Association between dopaminergic genetic variants, COMTrs4680 and DRD2rs1076560, and alcohol consumption and reward behaviors in non-dependent drinkers}

Nancy Elizabeth Ortega ${ }^{1}$, Bethany L. Stangl ${ }^{1}$,

Soundarya Soundararajan ${ }^{1}$, Shaliciana Burrell ${ }^{1}$, Hui Sun ${ }^{1}$, Melanie L. Schwandt ${ }^{1}$ and Vijay A. Ramchandani ${ }^{1}$

${ }^{1}$ National Institutes of Health

OBJECTIVES/SPECIFIC AIMS: The objective of this exploratory study is to evaluate the relationship between the individual genetic variants in COMTrs4680 and DRDrs1076560 and relevant alcohol use behaviors (i.e. alcohol consumption and reward processing behaviors) in non-dependent drinkers within experimentally controlled IV-ASA CAIS sessions. The overall goal of this study is to begin gathering data on the influence of individual genetic variants on alcohol consumption and other drinking-related behaviors. This will aid in the creation of a polygenic model of risk for AUD which will provide more insight into how the mesolimbic pathway is affected by alcohol use. METHODS/STUDY POPULATION: Study population: The sample included male and female non-dependent drinkers $(\mathrm{N}=149)$. Genotypes for functional polymorphisms in COMT (rs4680) and DRD2 (rs1076560) genes were determined for all subjects from blood samples obtained during screening. Alcohol consumption was assessed using the 90-day Timeline Followback Interviews (TLFB). Study population demographics: Self-reported gender (53.5\% identified as male); Self-reported race (61.2\% identified as white); Age ranged from 21-46 years old, with 22 years being the mode. Experiment: Free access (open-bar) intravenous alcohol self-administration (IV-ASA) using the computer-assisted alcohol infusion system (CAIS) paradigm; Subjects had the choice of pressing a button ad libitum for IV alcohol infusions during the session, neurobehavioral questionnaires were collected throughout the $2.5-\mathrm{hr}$ alcohol infusion session. Primary outcome measures included: Total Rewards, Peak breath alcohol concentration (BrAC) achieved, and Total Ethanol consumed. Statistical Analyses: Conducted using SPSS IBM Statistics Versions 1.0.0-2482; non-dependent drinkers were organized into two groups based on their genotypes, minor allele carriers and major allele homozygotes. Outcome measures were compared between genotype groups using analysis of variance or non-parametric Mann-Whitney U-test as appropriate. RESULTS/ ANTICIPATED RESULTS: -We expect the genetic makeup of the 
sample to be reflective of larger genome samples that are publically available (e.g. e!Ensembl) - Initial analysis for COMTrs4680 did not reveal significant effects on IV-ASA measures. Specifically, the majo DISCUSSION/SIGNIFICANCE OF IMPACT: Alcohol Use Disorder (AUD) affects millions of men and women globally. The heterogeneity within AUD individuals has made it difficult to identify biological and/or psychological factors that could be targeted for the development of treatments. By using the human laboratory model of free access IV-ASA, this study evaluated the relationship between dopaminergic genetic variants, COMTrs4680 and DRDrs1076560, and alcohol consumption in non-dependent drinkers within a controlled experimental environment. This study will begin to evaluate genetic and behavioral data that can be used to create a polygenic model of risk for AUD, which will provide more insight as to how the mesolimbic reward pathway is affected by alcohol use and contributes to risk for AUD.

\section{Association between Transfer of Children from the Emergency Department and Pediatric Readiness of the Hospital}

Monica Lieng ${ }^{1}$, Jennifer Rosenthal, Ilana Sigal, Parul Dayal, Sarah Haynes, Jamie Mouzoon, Amanda Favila and James Marcin ${ }^{1}$ UC Davis

OBJECTIVES/SPECIFIC AIMS: As pediatric care becomes more concentrated in large urban hospitals, smaller rural hospitals with reduced pediatric care capacity may opt to transfer pediatric patients to higher levels of care even if the patient has a condition that is manageable in a general ED. Up to $20-40 \%$ of pediatric transfers are considered avoidable, placing a burden on the patient, their family and the health care system. The aim of this study is to determine the association between pediatric readiness (as measured by the National Pediatric Readiness Project score) and risk of interfacility transfer. We hypothesized that emergency departments with higher measures of pediatric readiness would be less likely to transfer pediatric patients to another facility. METHODS/STUDY POPULATION: The most recent and complete National Pediatric Readiness Project data were from 300 California hospitals in 2012. These data include the overall pediatric readiness score, presence of inter-facility guidelines (written protocols on patients needing care not available at the hospital), presence of interfacility agreements (written agreements with other hospitals regarding transfer patients), and other variables on the facility's capacity to care for pediatric patients. We linked these hospital data with patient encounter data from the California Office of Statewide Health Planning and Development Emergency Department (ED) Database (OSHPD) using hospital name and zip code. To be eligible for the study, the patient must have a documented ED encounter and be less than 18 years old during the encounter. A patient was considered a transfer patient if they were transferred to a separate general hospital, children's hospital, federal health care facility, rehabilitation facility, Critical Access Hospital or psychiatric hospital. Chi-square tests and t-tests were used for descriptive statistics. For non-normal data, we used the nonparametric Kruskall-Wallis test. We also used logistic regression to compare the odds of transfer between comparison groups. Statistical analyses were conducted in the $\mathrm{R}$ environment version 3.4.4. RESULTS/ANTICIPATED RESULTS: In 2012, there were $2,604,723$ pediatric ED encounters, of which 10,966 resulted in a transfer $(0.4 \%)$. Transferred patients on average were older (15 vs. 5 years, $\mathrm{p}<0.001)$ and more likely to be female $(58.6 \%$ vs $46.6 \%$, $\mathrm{p}<0.001)$. The transfer group originated from hospitals with a lower median pediatric readiness score $(76.0$ vs $78.3, \mathrm{p}<0.001)$. Patients were less likely to be transferred if they were seen at a hospital with written guidelines with transfer protocols (OR 0.89, 95\% CI $0.83-0.95, \mathrm{p}<0.001)$. Patients were more likely to be transferred if they were seen at a hospital with written interfacility agreements with other hospitals (OR 1.17, 95\%CI 1.10-1.25, p < 0.001). We anticipate, that even with more sophisticated multilevel statistical models, pediatric readiness scores will remain associated with odds of transfer. DISCUSSION/SIGNIFICANCE OF IMPACT: These preliminary data suggest that hospitals with higher levels of pediatric readiness and written guidelines with transfer protocols are less likely to transfer pediatric patients. There may be actionable policy and procedural items that a hospital could enact to lower the rate of transfer patients. Future analyses will include more complex statistical modeling to adjust for confounders, will include inpatient data, and will compare the risk of potentially avoidable transfers between hospitals with varying levels of pediatric readiness.

3414 Association of blood pressure and biochemical knee
cartilage composition assessed by 2 relaxation time
measurements: Data from the Osteoarthritis Initiative Walid Ashmeik ${ }^{1}$, Gabby B. Joseph, Michael C. Nevitt, Nancy E. Lane, Charles E. McCulloch and Thomas Link

${ }^{1}$ University Of California, San Francisco

OBJECTIVES/SPECIFIC AIMS: The goal of this study was to investigate the associations of systolic blood pressure (SBP), diastolic blood pressure (DBP) and pulse pressure (PP) with knee articular cartilage composition using magnetic resonance imaging (MRI)-based T2 relaxation time measurements in study participants from the Osteoarthritis Initiative (OAI). METHODS/STUDY POPULATION: In this longitudinal study, 1,139 participants from the OAI, a multi-center, observational study of the evolution of knee $\mathrm{OA}$, were selected using the following inclusion criteria: right knee Kellgren Lawrence (KL) score (radiographic classification of OA severity) 0-2 indicating no to mild radiographic OA at baseline, no history of rheumatoid arthritis at baseline, available blood pressure measurements at baseline, available T2 measurements in at least three knee compartments at baseline and 48-month follow-up. Linear regression models were performed using standardized values for SBP, DBP and PP as primary predictors and change in cartilage T2 over 48 months, a measure of cartilage matrix quality and degeneration, as the primary outcome. PP was defined as SBP minus DBP. Change in superficial layer and deep layer cartilage T2, which reflect differences in the laminar organization of knee cartilage T2, were also included as outcomes. Statistical models were adjusted for common risk factors for knee OA (baseline age, sex, BMI, KL score) as well as number of currently used anti-hypertensive medications (AHM) reported at baseline. We included AHMs whose primary indication was the treatment of hypertension including beta blockers, angiotensin-converting enzyme (ACE) inhibitors, angiotensin II receptor blockers (ARB), thiazides, chlorthalidone, dihydropyridine calcium channel blockers (CCB) and aliskiren. All predictors, outcomes and covariates (except sex) were analyzed as continuous variables. We included interaction terms in the models to evaluate 